Model Reduction of Second-Order Systems.

Chahlaoui, Younes and Gallivan, Kyle A and Vandendorpe, Antoine and Van Dooren, Paul

2006

MIMS EPrint: 2008.11

Manchester Institute for Mathematical Sciences

School of Mathematics

The University of Manchester

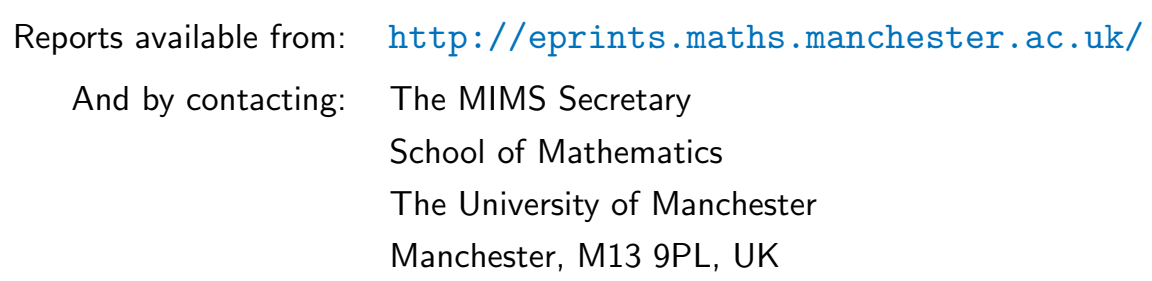

ISSN 1749-9097 
Y. Chahlaoui ${ }^{1}$, K. A. Gallivan ${ }^{1}$, A. Vandendorpe ${ }^{2}$, P. Van Dooren ${ }^{2}$

${ }^{1}$ School of Computational Science, Florida State University, Tallahassee, U.S.A. younes. chahlaoui $@ 1$ aposte. net, gallivan@ecsit. fsu. edu
CESAME, Université catholique de Louvain, Louvain-la-Neu

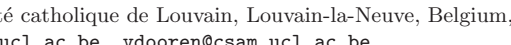

\subsection{Introduction}

In this chapter, the problem of constructing a reduced order system while preserving the second-order structure of the original system is discussed. Af model reduction techniques, two classes of second-order structure preserving model reduction techniques - Krylov subspace-based and SVD-based - are presented. For the Krylov techniques, conditions on the projectors that guarantee the reduced second-order system tangentially interpolates the original system at given frequencies are derived and an algorithm is described. For SVD-based techniques, a Second-Order Balanced Truncation method is derived from second-order Gramians.

Second-order systems arise naturally in many areas of engineering (see, for example, [Pre97, WJJ87]) and have the following form:

$$
\left\{\begin{aligned}
M \ddot{q}(t)+D \dot{q}(t)+S q(t) & =F^{\text {in }} u(t), \\
y(t) & =F^{\text {out }} q(t) .
\end{aligned}\right.
$$

We assume that $u(t) \in \mathbb{R}^{m}, y(t) \in \mathbb{R}^{p}, q(t) \in \mathbb{R}^{N}, F^{\text {in }} \in \mathbb{R}^{N \times m}, F^{\text {out }} \in$ with and stiffness matrices, $u(t)$ corresponds to the vector of erternal forces, $F^{i n}$ to the input distribution matrix, $y(t)$ to the output measurement vector, $F^{\text {out }}$ to the output measurement matrix, and $q(t)$ to the vector of internal generalized coordinates.

The transfer function associated with the system (6.1) links the outputs to the inputs in the Laplace domain and is given by

$$
R(s):=F^{o u t} P(s)^{-1} F^{i n},
$$

where

$$
P(s):=M s^{2}+D s+S
$$

is the characteristic polynomial matrix. The zeros of $\operatorname{det}(P(s))$ are also known as the characteristic frequencies of the system and play an important role in model reduction, e.g., the system is stable if these zeros lie in the open lef half plane.

Often, the original system is too large to allow the efficient solution of various control or simulation tasks. In order to address this problem, techniques that produce a reduced system of size $n \ll N$ that possesses the essential properties of the full order model have been developed. Such a reduced model can then be used effectively, e.g., in real-time, for controlling or simulating the foct

$$
\left\{\begin{aligned}
\hat{M} \ddot{q}(t)+\hat{D} \dot{\hat{q}}(t)+\hat{S} \hat{q}(t) & =\hat{F}^{i n} u(t) \\
\hat{y}(t) & =\hat{F}^{\text {out }} \hat{q}(t)
\end{aligned}\right.
$$

where $\hat{q}(t) \in \mathbb{R}^{n}, \hat{M}, \hat{D}, \hat{S} \in \mathbb{R}^{n \times n}, \hat{F}^{i n} \in \mathbb{R}^{n \times m}, \hat{F}^{\text {out }} \in \mathbb{R}^{p \times n}$, such that its transfer function is "close" to the original transfer function.

In contrast with second-order systems, first order systems can be represented as follows:

$$
\left\{\begin{array}{l}
\dot{x}(t)=A x(t)+B u(t) \\
y(t)=C x(t)
\end{array}\right.
$$

where again $u(t) \in \mathbb{R}^{m}, y(t) \in \mathbb{R}^{p}, x(t) \in \mathbb{R}^{N}, C \in \mathbb{R}^{p \times N}, A \in \mathbb{R}^{N \times N}$ and

The transfer function associated with the system (6.5) is given by

$$
R(s):=C\left(s I_{N}-A\right)^{-1} B .
$$

Second-order systems can be seen as a particular class of linear systems. Indeed, since the mass matrix $M$ is assumed to be invertible, we can rewrite the system (6.1) as follows

$$
\left\{\begin{array}{l}
\dot{x}(t)=\left[\begin{array}{cc}
0 & I_{N} \\
-S_{M} & -D_{M}
\end{array}\right] x(t)+\left[\begin{array}{c}
0 \\
F_{M}^{i n}
\end{array}\right] u(t) \\
y(t)=\left[\begin{array}{ll}
F_{M}^{\text {out }} & 0
\end{array}\right] x(t)
\end{array}\right.
$$

where the state $x(t)$ is $\left[q(t){ }^{T} \dot{q}(t)^{T}\right]^{T}$, and where $S_{M}=M^{-1} S, D_{M}=$
$M^{-1} D$, $F^{\text {in }}=M^{-1} F^{\text {in }}$, Fout $=F^{\text {out }}$, which is of the form $(6.5)$. We can thus rewrite the transfer function defined in (6.2) as

$$
R(s)=C\left(s I_{2 N}-A\right)^{-1} B
$$

with

$$
A:=\left[\begin{array}{cc}
0 & I_{N} \\
-S_{M} & -D_{M}
\end{array}\right] \quad, \quad B:=\left[\begin{array}{c}
0 \\
F_{M}^{i n}
\end{array}\right] \quad, \quad C:=\left[\begin{array}{ll}
F_{M}^{o u t} & 0
\end{array}\right] .
$$


Note that if the dimension of the state $q(t)$ of the original second-order system (6.1) is equal to $N$, the order of its corresponding linearized state space realization (6.9) (also called the McMillan degree of $R(s)$ if the state space realization $(C, A, B)$ is minimal) is equal to $2 N$.

A reduced model for the second-order system (6.1) could be produced by applying standard linear model reduction techniques to $(C, A, B)$ in $(6.9)$ to yield a small linear system $(C, A, B)$. Unfortunately, there is no guarante that the matrices defining the reduced system $(C, A, B)$ have the block structure necessary to preserve the second-order form of the original system. Such a guarantee requires the develop

This chapter is organized as follows. In Section 6.2, general results con6.3. a imple sufficient condition for constructing reduced order systems tion preserve the second-order structure is developed. Generalizations of Balanced Truncation and Krylov subspace-based methods that enforce this sufficient condition for second-order systems are presented in Sections 6.4 and 6.5, respectively. After some numerical experiments in Section 6.6, concluding remarks are given in Section 6.7 .

\subsection{Model Reduction of Linear Systems}

Most popular model reduction techniques for linear systems can be put in one of two categories [Ant05]: SVD-based and Krylov subspace-based techniques. Perhaps the most popular model reduction technique for linear systems is the Balanced Truncation method. This SVD-based technique has many advantages: the stability of the original system is preserved and there exists an priori global bound on the error between the original and the reduced system. The main drawback is that this technique cannot be applied to large-scale systems of order $N$, i.e., those systems where $O\left(N^{3}\right)$ computations is an unacceptably large cost. On the other hand, Krylov subspace-based techniques that are based on imposing moment matching conditions between the original and the reduced transfer function, such as rational/tangential interpolation methods, can be applied to large-scale systems but do not provide globa
bounds and depend significantly on the choice of certain parameters.

In this section, we present an overview of examples of each category applied then strictly proper ie lim $R(s)=0$. Since $M$ is invertible, the transfor

\subsubsection{Balanced Truncation}

If $A$ is stable, then the system (6.5) is also a linear (convolution) operato mapning squire integrable inputs $u(t) \in \mathcal{L}[-\infty,+\infty]$ to square integrable outputs $y(t) \in \mathcal{L}_{2}[-\infty,+\infty]$. Following the development in [CLVV05], we recall the concept of a dual operator to discuss the Balanced Truncation method.

Definition 6.2.1. Let $L$ be a linear operator acting from a Hilbert space $U$ to a Hilbert space $Y$ equipped respectively with the inner products $<,>_{U}$ and from $Y$ to $U$ such that $\langle L u, y\rangle_{Y}=\left\langle u, L^{*} y\right\rangle_{U}$ for all $y \in Y$ and all $u \in U$.

It is easily verified that the transfer function associated with the dual operato of (6.6) is $B^{T}\left(s I_{N}-A^{T}\right)^{-1} C^{T}$, (see [ZDG95]),

Now consider the input/output behavior of the system (6.5). If we apply an input $u(t) \in \mathcal{L}_{2}[-\infty, 0]$ to the system for $t<0$, the position of the state at time $t=0$, assuming the zero initial condition $x(-\infty)=0$, is equal to

$$
x(0)=\int_{-\infty}^{0} e^{-A t} B u(t) d t:=\mathcal{C}_{o} u(t) .
$$

If a zero input is applied to the system for $t>0$, then for all $t \geq 0$, the output $y(t) \in \mathcal{L}_{2}[0,+\infty]$ of the system (6.5) is equal to

$$
y(t)=C e^{A t} x(0):=\mathcal{O}_{b} x(0) .
$$

So the mapping of past inputs to future outputs is characterized by two operators - the so-called controllability operator $\mathcal{C}_{o}: \mathcal{L}_{2}[-\infty, 0] \mapsto \mathbb{R}^{n}$ $\mathcal{O}_{b}: \mathbb{R}^{n} \mapsto \mathcal{L}_{2}[0,+\infty]$ (mapping the present state to future outputs $y(t)$ ).

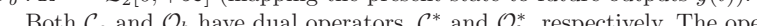

ors and their duals are related by two fundamental matrices associated with the linear system (6.5). These are the "controllability Cramian" $\mathcal{P}$ and the "observability Gramian" $\mathcal{Q}$. If $A$ is stable, they are the unique solutions of the Lyapunov equations:

$$
A \mathcal{P}+\mathcal{P} A^{T}+B B^{T}=0 \quad, \quad A^{T} \mathcal{Q}+\mathcal{Q} A+C^{T} C=0 .
$$

It follows that $\mathcal{C}_{o}$ and $\mathcal{O}_{b}$ are related to their dual operators by the identitie $\mathrm{P}=\mathcal{C}_{o}^{*} \mathcal{C}_{o}$ and $\mathcal{Q}=\mathcal{O}_{b} \mathcal{O}_{b}^{*}$ [ZDG95].

interpretation of the Gramians results from two opt mization problems. Let

$$
J(v(t), a, b):=\int_{a}^{b} v(t)^{T} v(t) d t
$$

that (see [ZDG95])

$$
\min _{\mathcal{C}_{\circ} u(t)=x_{0}} J(u(t),-\infty, 0)=x_{0}^{T} \mathcal{P}^{-1} x_{0},
$$


and, symmetrically, we have the dual property

$$
\min _{\mathcal{O}_{b}^{*} y(t)=x_{0}} J(y(t),-\infty, 0)=x_{0}^{T} \mathcal{Q}^{-1} x_{0} .
$$

Two algebraic properties of Gramians $\mathcal{P}$ and $\mathcal{Q}$ are essential to the development of Balanced Truncation. First, under a coordinate transformation $x(t)=T \bar{x}(t), \bar{C}, \bar{A}, \bar{B}):=\left(C T, T^{-1} A T, T^{-1} B\right)$ consesponding to the state-space realization $(\bar{C}, \bar{A}, \bar{B}):=\left(C T, T^{-1} A T, T^{-1} B\right)$ undergo the following (so-called contragradient) transformation:

$$
\overline{\mathcal{P}}=T^{-1} \mathcal{P} T^{-T}, \quad \overline{\mathcal{Q}}=T^{T} \mathcal{Q} T .
$$

This implies that the eigenvalues of the product $\overline{\mathcal{P}} \overline{\mathcal{Q}}=T^{-1} \mathcal{P} \mathcal{Q} T$ depend only on the transfer function $R(s)$ and not on a particular choice of statespace realization. It implies also that there exists a state-space realizatio $\left(C_{b a l}, A_{b a l}, B_{b a l}\right)$ of $R(s)$ such that the corresponding Gramians are equal and diagonal $\overline{\mathcal{P}}=\overline{\mathcal{Q}}=\Sigma$ [ZDG95]

Second, because the Gramians appear in the solutions of the optimization problems (6.11) and (6.12), they give information about the energy that goes through the system, more specifically, about the distribution of this energy among the state variables. The smaller $x_{0}^{2} \mathrm{p}^{-1} x_{0}$ is, the more "controllable" the state $x_{0}$ is, since it can be reached with a input of small energy. By duality, the smaller $x_{0}^{T} \mathcal{Q}^{-1} x_{0}$ is, the more "observable" the state $x_{0}$ is. Thus when both Gramians are equal and diagonal, the order of magnitude of diagonal value of the product $\mathcal{P} \mathcal{Q}$ is a good measure for the influence of the correspor inputs $u(t) \in \mathcal{L}_{2}[-\infty, 0]$ to

Given a transfer function $R(s)$, the Balanced Truncation model reduction method consists of finding a state-space realization $\left(C_{b a l}, A_{b a l}, B_{b a l}\right)$ of $R(s)$ of the system) and then constructing the reduced model by keeping the states corresponding to the largest eigenvalues of the product $\mathcal{P} \mathcal{Q}$ and discardin the others. In other words the balanced truncation technique chooses $Z$ and $V$ such that $Z^{T} V=I$, and

$$
\left\{\begin{array}{l}
\mathcal{P} \mathcal{Q} V=V \Lambda_{+} \\
\mathcal{Q P} Z=Z \Lambda_{+}
\end{array}\right.
$$

where $\Lambda_{+}$is a square diagonal matrix containing the largest eigenvalues of $\mathcal{P Q}$. A state-space realization of the reduced transfer function is given by $\left(C V, Z^{T} A V, Z^{T} B\right)$. The idea of the balanced truncation technique thus consists in keeping those states that are most controllable and observable according to the Gramians defined in (6.10).

Finally, we note that Balanced Truncation can be related to the Hankel , we naps inputs to the future outputs and is defined as
$\mathcal{H}:=\mathcal{O}_{b} \mathcal{C}_{o}$. Since $\mathcal{P} \mathcal{Q}=\mathcal{C}_{o} \mathcal{C}_{o}^{*} \mathcal{O}_{b}^{*} \mathcal{O}_{b}$ and $\mathcal{Q P}=\mathcal{O}_{b}^{*} \mathcal{O}_{b} \mathcal{C}_{o} \mathcal{C}_{o}^{*}$, the dominant eigenspaces $\mathcal{V}$ of $\mathcal{P Q}$ and $\mathcal{Z}$ of $\mathcal{Q P}$ are linked with the dominant eigenspaces $\mathcal{X}$ of $\mathcal{H H}^{*}$ and $\mathcal{Y}$ of $\mathcal{H}^{*} \mathcal{H}$ via the equalities $\mathcal{X}=\mathcal{O}_{b} \mathcal{V}$ and $\mathcal{Y}=\mathcal{C}_{o}^{*} \mathcal{Z}$. Therefore, projecting onto the spaces $\mathcal{V}$ and $\mathcal{Z}$ also approximates the Hankel map $\mathcal{H}$ well. We refer the reader to [ZDG95], for a more detailed study and discussion of the Balanced Truncation method.

Unfortunately, the Balanced Truncation method cannot be applied directly to the state-space realization $(C, A, B)(6.7)$ of the second-order system without destroying its second-order structure in the reduced realization, that, due to its dependence Balanced Truncetion methot cannot be appliet, as described to large systems. Recent work by Antoulas and Sorenen considers this problescale describes an A ppoximate Balanced Truncation appreach for lorgecale liner systems [SA02].

\subsubsection{Krylov Subspace-Based Model Reduction}

The Krylov subspace-based model reduction methods have been developed in order to produce reduced order models of large-scale linear systems efficiently and stably via projection onto subspaces that satisfy specific conditions. These conditions are based on requiring the reduced order transfer function to match selected moments of the transfer function $R(s)$ of the original system.

A rational matrix function $R(s)$ is said to be $O(\lambda-s)^{k}$ in $s$ with $k \in \mathbb{Z}$ if its Taylor expansion about the point $\lambda$ can be written as

$$
R(s)=O(\lambda-s)^{k} \Longleftrightarrow R(s)=\sum_{i=k}^{+\infty} R_{i}(\lambda-s)^{i},
$$

where the coefficients $R_{i}$ are constant matrices. If $R_{k} \neq 0$, then we say that $R(s)=\Theta(\lambda-s)^{k}$. As a consequence, if $R(s)=\Theta(\lambda-s)^{k}$ and $k$ is strictly negative, then $\lambda$ is a pole of $R(s)$ and if $k$ is strictly positive, then $\lambda$ is a zero of $R(s)$. Analogously, we say that $R(s)$ is $O\left(s^{-1}\right)^{k}$ if the following condition is satisfied:

$$
R(s)=O\left(s^{-1}\right)^{k} \Longleftrightarrow R(s)=\sum_{i=k}^{+\infty} R_{i} s^{-i},
$$

where the coefficients $R_{i}$ are constant matrices. It should be stressed that, in general, $R(s)$ being $O(s)^{-k}$ is not equivalent to $R(s)$ being $O\left(s^{-1}\right)^{k}$.

\section{Rational Interpolation}

Krylov subspaces play an important role in the development of these methods and are defined as follows: 
If $A$ is stable, $R(s)$ expanded about infinity gives

$$
R(s)=C\left(s I_{N}-A\right)^{-1} B=\sum_{i=0}^{\infty} C A^{i} B s^{-i-1}:=\sum_{i=0}^{\infty} R_{i}^{(\infty)} s^{-i-1},
$$

where the coefficients $R_{i}^{\infty}$ are called the Markov parameters of the system. One intuitive way to approximate $R(s)$ is to construct a transfer function $\hat{R}(s)$ of McMillan degree $n \ll N$

$$
\hat{R}(s):=\hat{C}\left(s I_{n}-\hat{A}\right)^{-1} \hat{B}:=\sum_{i=1}^{\infty} \hat{R}_{i}^{(\infty)} s^{-i}
$$

such that $\hat{R}_{i}^{(\infty)}=R_{i}^{(\infty)}$ for $1 \leq i \leq r$, where $r$ is as large as possible and is generically equal to $2 n$. The resulting reduced transfer function $\hat{R}(s)$ generally
approximates quite well the original transfer function for large values of $s$.

If a good approximation for low frequencies is desired, one can construct transfer function

$$
\hat{R}(s)=\hat{C}\left(s I_{n}-\hat{A}\right)^{-1} \hat{B}=\sum_{k=0}^{\infty} \hat{R}_{k}^{(\lambda)}(\lambda-s)^{k}
$$

such that

$$
\hat{R}_{k}^{(\lambda)}=R_{k}^{(\lambda)} \quad \text { for } 1 \leq k \leq K,
$$

with

$$
R_{k}^{(\lambda)}:=C\left(\lambda I_{N}-A\right)^{-k} B, \quad \hat{R}_{k}^{(\lambda)}:=\hat{C}\left(\lambda I_{n}-\hat{A}\right)^{-k} \hat{B} .
$$

In short, (6.18) can be rewritten as follows:

$$
R(s)-\hat{R}(s)=O(\lambda-s)^{K} .
$$

More generally, one can choose a transfer function $\hat{R}(s)$ that interpolates $R(s)$ at several points in the complex plane, up to several orders. The main results concerning this problem for $\mathrm{M}$.
summarized in the following theorem.

Theorem 6.2.3. Let the original system be

$$
R(s):=C\left(s I_{N}-A\right)^{-1} B,
$$

and the reduced system be

$$
\hat{R}(s):=C V\left(Z^{T}\left(s I_{N}-A\right) V\right)^{-1} Z^{T} B,
$$

with $Z^{T} V=I_{n}$. If

$$
\bigcup_{k=1}^{K} \mathcal{K}_{b_{k}}\left(\left(\lambda_{k} I-A\right)^{-1},\left(\lambda_{k} I-A\right)^{-1} B\right) \subseteq \operatorname{Im}(V)
$$

and

$$
\bigcup_{k=1}^{K} \mathcal{K}_{c_{k}}\left(\left(\lambda_{k} I-A\right)^{-T},\left(\lambda_{k} I-A\right)^{-T} C^{T}\right) \subseteq \operatorname{Im}(Z)
$$

where the interpolation points $\lambda_{k}$ are chosen such that the matrices $\lambda_{k} I_{N}-A$ are invertible $\forall k \in\{1, \ldots, K\}$ then the moments of the systems (6.19) and (6.20) at the points $\lambda_{k}$ satisfy

$$
R(s)-\hat{R}(s)=O\left(s-\lambda_{k}\right)^{b_{k}+c_{k}},
$$

prove.

For a proof, see [dVS87] and [Gri97]. A proof for MIMO generalized state space systems is given in [GVV04b].

Matching Markov parameters, i.e., $\lambda=\infty$, is known as partial realization When $\lambda=0$, the corresponding problem is known as Padé approximation. If $\lambda$ takes a finite number of points $\lambda_{i}$, it is called a multi-point Padé approximation. In the general case, the problem is known as rational interpolation. Rational interpolation generally results in a good approximation of the original transfer function in a region near the expansion points (and increasing other frequencies (see for instance [Ant05]).

The advantage of these moment matching methods is that they can be implemented in a numerically stable and efficient way for large-scale system. with sparse coefficient matrices (see for example [GVV04b] and [Gri97]). Also, the local approximation property means that good approximations can be achieved in specific regions over a wide dynamic range, typically at the cost of a larger global error. This requires however, that the interpolation points and their corresponding order of approximation must be specified. For some applications, the user may have such information but for blackbox library software a heuristic automatic selection strategy is needed (see [Gri97]) and the design of such a strategy is still an open question. The other main drawback is the lack of an error bound on the global quality of the approximation, e.g., the $H_{\infty}$ reduced order model that may help in selecting points [CGV04]

One could apply these methods to the state space mellats second-order transfer function. Unfortunately, if the methods are (6.9) in the forms described, the resulting reduced order transfer function will menerically not be in second-order form. An approach to maintain second-order forn discussed in Section 6.5 
The Krylov subspace-based methods that produce reduced order models based on rational interpolation can be applied to MIMO systems efficiently as long as the For MINO systens where $m$ and $p$ are too large, a more general tangential of imposing interpolation condition of the form $R\left(\lambda_{i}\right)=\hat{R}\left(\lambda_{i}\right)$, one could be interested, for example, in only imposing interpolation conditions of the lowing form:

$\hat{R}\left(\lambda_{i}\right) x_{i}=R\left(\lambda_{i}\right) x_{i} \quad, \quad y_{i} \hat{R}\left(\lambda_{i+n}\right)=y_{i} R\left(\lambda_{i+n}\right), \quad 1 \leq i \leq n, \quad(6.24)$

where the $n$ column vectors $x_{i}$ are called the right interpolation directions and the $n$ row vectors $y_{i}$ are called the left interpolation directions. As with rational interpolation, higher order tangential interpolation conditions can be imposed at each point to improve the approximation.

Stable and efficient methods for tangential interpolation of MIMO systems can be developed using theorems and techniques similar to those used for Krylov subspace-based rational interpolation. However, the problem of constructing a reduced transfer function that satisfies a set of tangential interpo-

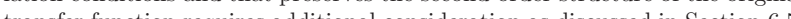

\subsection{Second-Order Structure Preserving Model Reduction}

In this section, a simple sufficient condition for obtaining a second-order reduced system from a second-order system is presented. The following result can be found in a slightly different form in [CLVV05]

Lemma 6.3.1. Let $(C, A, B)$ be the state space realization defined in (6.9). If one projects such a state space realization with $2 N \times 2 n$ bloc diagonal matrice

$$
\bar{Z}:=\left[\begin{array}{cc}
Z_{1} & 0 \\
0 & Z_{2}
\end{array}\right], \quad \bar{V}:=\left[\begin{array}{cc}
V_{1} & 0 \\
0 & V_{2}
\end{array}\right], \quad \bar{Z}^{T} \bar{V}=I_{2 n},
$$

where $Z_{1}, V_{1}, Z_{2}, V_{2} \in \mathbb{C}^{N \times n}$, then the reduced transfer function

$$
\hat{R}(s):=C \bar{V}\left(\bar{Z}^{T}\left(s I_{2 N}-A\right) \bar{V}\right)^{-1} \bar{Z}^{T} B
$$

is a second-order transfer function, provided the matrix $Z_{1}^{T} V_{2}$ is invertible.

Proof. First, notice that the transfer function does not change under any similarity transformation of the system matrices. Let us consider the similarity
transformation $M \in \mathbb{C}^{2 n \times 2 n}$ such that

with $X, Y \in \mathbb{C}^{n \times n}$ verifying

$$
M:=\left[\begin{array}{ll}
X & \\
& Y
\end{array}\right],
$$

$$
X^{-1}\left(Z_{1}^{T} V_{2}\right) Y=I_{n} .
$$

From the preceding results,

$\hat{R}(s):=C \bar{V} M\left(M^{-1} \bar{Z}^{T}\left(s I_{2 N}-A\right) \bar{V} M\right)^{-1} M^{-1} \bar{Z}^{T} B$

$=F_{M}^{o u t} V_{1} X\left(s^{2} I_{n}+s Y^{-1} Z_{2}^{T} D_{M} V_{2} Y+Y^{-1} Z_{2}^{T} S_{M} V_{1} X\right)^{-1} Y^{-1} Z_{2}^{T} F_{M}^{i n}$.

This is clearly a second-order transfer function.

\subsection{Second-Order Balanced Truncation}

The earliest balanced truncation technique for second-order systems known to the authors is described in [MS96]. Based on this work, an alternative technique was developed in [CLVV05]. In this section an overview of the latter method, called SOBT (Second-Order Balanced Truncation), is given.

The first step in the development of SOBT, based on a balance and trumcate process similar to that discussed in Section 6.2.1, involves the definition of two pairs of $N \times N$ Gramians ("second-order Gramians") that change according to contragradient transformations, and that have some energetic interpretation. The first pair $\left(\mathcal{P}_{p o s}, \mathcal{Q}_{p o s}\right)$ corresponds to an energy optimization problem depending only on the positions $q(t)$ and not on the velocities $q(t)$. Reciprocally, the second pair $\left(\mathcal{P}_{\text {vel }}, \mathcal{Q}_{\text {vel }}\right)$ correspond to an optimization problem depending only on the velocities $\dot{q}(t)$ and not the on the positions $q(t)$. By analogy to the first order case, the Gramians $\mathcal{Q}_{\text {pos }}$ and $\mathcal{Q}_{v e l}$ are define from the dual systems. Given the Gramians, a balancing step in the method is defined by transforming to a coordinate system in which the second-order Their diagonal values enable us to identify the important positions $=\Sigma_{\text {vel }}$. important veloties, i.e those with (hop fllly) hare effect on the $\mathrm{I} / \mathrm{O}$ maf Once identified, the reduced second-order model follows by trunction of al vishes identified as inportant.

of the contributo the I/O map, consider an optimization problem netocities) with respect the second-order system (see [MS96]) of the form

$\min _{\dot{q}_{0} \in \mathbb{R}^{\mathrm{n}}} \min _{u(t)} J(u(t),-\infty, 0)$,

subject to 
$\ddot{q}(t)+D_{M} \dot{q}(t)+S_{M} q(t)=F_{M}^{i n} u(t), q(0)=q_{0}$.

One easily sees that the optimum is $q_{0}^{T} \mathcal{P}_{11}{ }^{-1} q_{0}$, where $\mathcal{P}_{11}$ is the $N \times N$ left upper block of the controllability Gramian $\mathcal{P}$ satisfying equation (6.10) with $(C, A, B)$ given in (6.9). Starting with (6.11) we must solve

$$
\min _{\dot{q}_{0} \in \mathbb{R}^{\mathrm{R}}} J_{q_{0}}\left(\dot{q}_{0}\right)=\left[q_{0}^{T} \dot{q}_{0}^{T}\right] \mathcal{P}^{-1}\left[\begin{array}{c}
q_{0} \\
\dot{q}_{0}
\end{array}\right]
$$

Partitioning $\mathcal{P}^{-1}$ as follows

$$
\mathcal{P}^{-1}=\left[\begin{array}{ll}
R_{1} & R_{2} \\
R_{2}^{T} & R_{3}
\end{array}\right]
$$

and annihilating the gradient of $J_{q_{0}}\left(\dot{q}_{0}\right)$ gives the relation $\dot{q}_{0}=-R_{3}^{-1} R_{2}^{T} q_{0}$. The value of $J_{q_{0}}$ at this point is $q_{0}^{T}\left(R_{1}-R_{2} R_{3}^{-1} R_{2}^{T}\right) q_{0}$. This is simply the Schur complement of $R_{3}$ which is $\mathcal{P}_{11}^{-1}$. Similarly, the solution of the dual problem corresponds to $q_{0}^{T} \mathcal{Q}_{11}{ }^{-1} q_{0}$, where $\mathcal{Q}_{11}$ is the $N \times N$ left upper block the observability Gramian $\mathcal{Q}(6.10)$.

Note that the transfer function is seen as a linear operator acting between two Hilbert spaces. The dual of such an operator is defined in Definition 6.2.1. It follows that the dual of a second-order transfer function might not the energy transfer interpretation between the inputs, the outputs, the initi positions and velocities is important. Under the change of coordinates $g(t)=$ $T \bar{q}(t)$, it is easy to verify that this pair of Cramians undergoes a contragradient transformation:

$$
\left(\overline{\mathcal{P}}_{11}, \overline{\mathcal{Q}}_{11}\right)=\left(T^{-1} \mathcal{P}_{11} T^{-T}, T^{T} \mathcal{Q}_{11} T\right) .
$$

This implies that there exists a new coordinate system such that both $\mathcal{P}_{11}$ and $\mathcal{Q}_{11}$ are equal and diagonal. Their energetic interpretation is seen by con to reach the aiven position . mized. Henee, these Gra among the positions.$$
\text { A pair of second-ord }
$$

perato ities with of the I/O map can be defined analogously. The associated optimization problem is

$$
\min _{q_{0} \in \mathbb{R}^{n}} \min _{u(t)} J(u(t),-\infty, 0)
$$

subject to

$$
\ddot{q}(t)+D_{M} \dot{q}(t)+S_{M} q(t)=F_{M}^{i n} u(t), \quad \dot{q}(0)=\dot{q}_{0} .
$$
Following the same reasoning as before for the optimization problem (6.25) right lower block of $\mathcal{P}$. The solution of the dual problem is $\dot{q}_{0}^{T} \mathcal{Q}_{22}{ }^{-1} \dot{q}_{0}$, where $\mathcal{Q}_{22}$ is the $N \times N$ right lower block of $\mathcal{Q}$. As before, under the change of coordinates $q(t)=T \bar{q}(t)$ one can check that this pair of Gramians undergoes a contragradient transformation and that the energetic interpretation is given by considering the underlying optimization problem. In (6.26), the energy necessary to reach the given velocity $q_{0}$ over all past inputs and initial position is minimized. Hence, these Gramians describe the distribution of the $\mathrm{I} / \mathrm{O}$ energy among the velocities.

Given the interpretation above these second-order Gramians are good canruncating. Therefore, we choose:

$\left(\mathcal{P}_{\text {pos }}, \mathcal{Q}_{\text {pos }}\right)=\left(\mathcal{P}_{11}, \mathcal{Q}_{11}\right)$ and $\left(\mathcal{P}_{v e l}, \mathcal{Q}_{v e l}\right)=\left(\mathcal{P}_{22}, \mathcal{Q}_{22}\right)$

It is not possible to balance both pairs of second-order Gramians at the same time with a single change of coordinates of the type $q(t)=T \bar{q}(t)$. A change of coordinates is required for both positions and velocities (unlike the approach in [MS96]). Therefore, we work in a state-space context, starting with the system (6.9). The SOBT method, therefore, first computes both pairs of second-order Gramians, $\left(\mathcal{P}_{\text {pos }}, \mathcal{Q}_{\text {pos }}\right)$ and $\left(\mathcal{P}_{\text {vel }}, \mathcal{Q}_{\text {vel }}\right)$. Given the Gramians, the contragradient transformations that make $\mathcal{P}_{\text {pos }}=\mathcal{Q}_{\text {pos }}=\Lambda_{p o s}$ and $\mathcal{P}_{\text {vel }}=\mathcal{Q}_{\text {vel }}=\Lambda_{\text {vel }}$, where $\Lambda_{\text {pos }}$ and $\Lambda_{\text {vel }}$ are positive definite diagonal matrices, are computed. Finally, truncate the positions corresponding to the smallest eigenvalues of $\Lambda_{p o s}$ and the velocities corresponding to the smallest eigenvalues of $\Lambda_{v e l}$

At present, there exists no a priori global error bound for SOBT and the stability of the reduced system is not guaranteed. Nevertheless, SOBT yield good rumercal results, providing reduced transier functions with approxima-

\subsection{Second-Order Structure Preserving Krylov}

\section{Techniques}

The Krylov subspace-based methods discussed in Section 6.2.2 do not preserve second-order structure when applied to the linear system (6.9). It is possible produce a theco to satisfy the constraint presented in Section 6.3 and thereby Krylov subspace-based method for second-order systems [SC91]. The simple technique constructs, via projection, a second-order reduced transfer function that matches the Markov parameters $(\lambda=\infty)$ of the original transfer function. The limitation of the technique when applied to a complex interpolation point is also discussed. Section 6.5.2, addresses this limitation using a generalization that allows multipoint rational interpolation. Finally, the problem of secondorder structure preserving tangential interpolation is solved in 6.5.3. 
Su and Craig proposed a Krylov subspace-based projection method that preserves second-order structure while matching the Markov parameters of the the right Krylow subsece corresporing to iiterpolation at $\lambda=\infty$ for the system (6.9) has the form

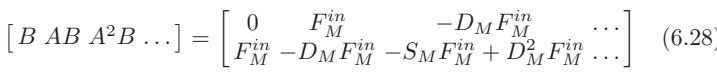

$$
\begin{aligned}
& =\left[\begin{array}{cccc}
0 & Q_{v, 0} & Q_{v, 1} & \ldots \\
Q_{v, 0} & Q_{v, 1} & Q_{v, 2} & \ldots
\end{array}\right] .
\end{aligned}
$$

and that if we write

$$
\mathcal{K}_{k}(A, B)=\left[\begin{array}{c}
V_{1} \\
V_{2}
\end{array}\right],
$$

it follows that

$$
\operatorname{Im}\left(V_{1}\right) \subseteq \operatorname{Im}\left(V_{2}\right) .
$$

So by projecting the state space realization (6.9) with

$$
\bar{V}:=\left[\begin{array}{cc}
V_{2} & 0 \\
0 & V_{2}
\end{array}\right], \quad \bar{Z}:=\left[\begin{array}{ll}
Z & 0 \\
0 & Z
\end{array}\right]
$$

such that $Z^{T} V_{2}=I_{n}$, we obtain an interpolating second-order transfer function of the form

$$
\hat{R}(s)=F_{M}^{o u t} V_{2}\left(Z^{T}\left(s^{2} I_{N}+s D_{M}+S_{M}\right)^{-1} V_{2}\right) Z^{T} F_{M}^{i n} .
$$

Hence, a second-order system with the same $n$ first Markov parameters as the original second-order system can be constructed by projecting with $Q_{v, 0} \ldots, Q_{v, n-1}$. Since $\mathcal{K}_{n}(A, B) \subseteq \bar{V}$, it follows from Theorem 6.2.3 that the first $n$ Markov parameters of $R(s)$ and $\hat{R}(s)$ are equal.

If we apply the construction for any interpolation point $\lambda \in \mathbb{C}$, the correponding right Krylov space is such that

$$
\mathcal{K}_{k}\left((\lambda I-A)^{-1},(\lambda I-A)^{-1} B\right)=\operatorname{Im}\left[\begin{array}{l}
V_{1} \\
V_{2}
\end{array}\right],
$$

with $A$ and $B$ defined in (6.9) and

$$
\operatorname{Im}\left(V_{1}\right) \subseteq \operatorname{Im}\left(V_{2}\right) .
$$

Unfortunately, a similar statement can not be made for the left Krylov subspaces $\mathcal{K}_{k}\left((\lambda I-A)^{-T},(\lambda I-A)^{-T} C^{T}\right)$. This implies that when the secondcomplex plane by projecting as in (6.30), only $n$ interpolation conditions can be imposed for a reduced second-order system of McMillan degree $2 n$.

\subsubsection{Second-Order Rational Interpolation}

The projection technique of Su and Craig has been generalized independently by several authors (see [VV04, BS04] and also Chapter 7 and Chapter 8) to solve the rational interolation pioble that produces a second-order transter function of order n, i.e., of McNillan degree $2 n, h(s)$, that interpolates $R(s)$ at the conditions that determine the projections are given in Theorem 6.5.1 and the associated algorithm is presented. By combining the results of Sections 6.2 and 6.3, the following theorem can be proven.

Theorem 6.5.1. Let $R(s):=F_{M}^{\text {out }}\left(s^{2} I_{N}+D_{M} s+S_{M}\right)^{-1} F_{M}^{i n}=C\left(s I_{2 N}-\right.$ $A)^{-1} B$, with

$$
A:=\left[\begin{array}{cc}
0 & I_{N} \\
-S_{M} & -D_{M}
\end{array}\right], \quad B:=\left[\begin{array}{c}
0 \\
F_{M}^{\text {in }}
\end{array}\right], \quad C:=\left[F_{M}^{\text {out }} 0\right],
$$

be a second-order transfer function of McMillan degree $2 N$, i.e. $S_{M}, D_{M} \in$ $\left.\mathbb{C}^{N \times N}\right)$. Let $Z, V \in \mathbb{C}^{2 N \times n}$ be defined as

$$
V:=\left[\begin{array}{l}
V_{1} \\
V_{2}
\end{array}\right] \quad, \quad Z:=\left[\begin{array}{l}
Z_{1} \\
Z_{2}
\end{array}\right],
$$

with $V_{1}, V_{2}, Z_{1}$ and $Z_{2} \in \mathbb{C}^{N \times n}$ such that

$$
Z_{1}^{T} V_{1}=Z_{2}^{T} V_{2}=I_{n}
$$

Let us define the $2 N \times 2 n$ projecting matrices

$$
\bar{V}:=\left[\begin{array}{cc}
V_{1} & 0 \\
0 & V_{2}
\end{array}\right] \quad, \quad \bar{Z}:=\left[\begin{array}{cc}
Z_{1} & 0 \\
0 & Z_{2}
\end{array}\right] .
$$

Define the second-order transfer function $\hat{R}(s)$ of order $n$ (and of McMillan degree $2 n$ ) by

$$
\hat{R}(s):=C \bar{V}\left(\bar{Z}^{T}\left(s I_{2 N}-A\right) \bar{V}\right)^{-1} \bar{Z}^{T} B
$$$$
:=\hat{C}\left(s I_{2 n}-\hat{A}\right)^{-1} \hat{B}
$$

If

$$
\bigcup_{k=1}^{K} \mathcal{K}_{b_{k}}\left(\left(\lambda_{k} I_{2 N}-A\right)^{-1},\left(\lambda_{k} I_{2 N}-A\right)^{-1} B\right) \subseteq \operatorname{Im}(V)
$$

and

$$
\bigcup_{k=1}^{K} \mathcal{K}_{c_{k}}\left(\left(\lambda_{k} I_{2 N}-A\right)^{-T},\left(\lambda_{k} I_{2 N}-A\right)^{-T} C^{T}\right) \subseteq \operatorname{Im}(Z)
$$


where the interpolation points $\lambda_{k}$ are chosen such that the matrices $\lambda_{k} I_{2 N}-A$ are invertible $\forall k \in\{1, \ldots, K\}$ then, if the matrix $Z_{1}^{T} V_{2}$ is invertible,

$$
R(s)-\hat{R}(s)=O\left(s-\lambda_{k}\right)^{b_{k}+c_{k}}
$$

for the finite points $\lambda_{k}$, provided these moments exist, i.e. provided the matrices $\lambda_{k} I_{2 n}-\hat{A}$ are invertible and

$$
R(s)-\hat{R}(s)=O\left(s^{-1}\right)^{b_{k}+c_{k}}
$$

if $\lambda_{k}=\infty$.

Proof. Clearly, $\bar{Z}^{T} \bar{V}=I_{2 n}$. The second-order structure of $\hat{R}(s)$ follows from Lemma 6.3.1. It is clear that

$$
\operatorname{Im}(V) \subset \operatorname{Im}(\bar{V}) \quad, \quad \operatorname{Im}(Z) \subset \operatorname{Im}(\bar{Z}) .
$$

The interpolation conditions are then satisfied because of Theorem 6.2.3.

The form of the projectors allows the development of an algorithm similar to the Rational Krylov family of algorithms for first order systems [Gri97] The algorither shown below, $\hat{R}(s)$, thecond-order transter function of order $n$, i.e. $\lambda_{1}$ up to $\lambda_{2 n}$, i.e.,
points

$$
R(s)-\hat{R}(s)=O\left(\lambda_{i}-s\right) \quad \text { for } 1 \leq i \leq 2 n .
$$

We assume for simplicity that the interpolation points are finite, distinct and not poles of $R(s)$. The algorithm is easily modified to impose higher order conditions at the interpolation points.

Algorithm 1 1. Construct $Z$ and $V$ such that

$$
\begin{aligned}
V & =\left[\left(\lambda_{1} I_{2 N}-A\right)^{-1} B \ldots\left(\lambda_{n} I_{2 N}-A\right)^{-1} B\right]=\left[\begin{array}{l}
V_{1} \\
V_{2}
\end{array}\right] \\
Z^{T} & =\left[\begin{array}{c}
C\left(\lambda_{n+1} I_{2 N}-A\right)^{-1} \\
\vdots \\
C\left(\lambda_{2 n} I_{2 N}-A\right)^{-1}
\end{array}\right]=\left[\begin{array}{ll}
Z_{1}^{T} & Z_{2}^{T}
\end{array}\right],
\end{aligned}
$$

where $V_{1}, V_{2} \in \mathbb{C}^{N \times n}$ are the first $N$ rows and the last $N$ rows of $V$ respectively and $Z_{1}, Z_{2} \in \mathbb{C}^{N \times n}$ are the first $N$ rows and the last $N$ rows $N^{T} Z^{T} V_{1} M_{1}=N_{2}^{T} Z^{T} V_{2} M_{2}=I_{2}$. $1 Z_{1} V_{1}=N_{2}$

$$
\bar{V}:=\left[\begin{array}{ll}
V_{1} M_{1} & \\
& V_{2} M_{2}
\end{array}\right] \quad, \quad \bar{Z}:=\left[\begin{array}{ll}
Z_{1} N_{1} & \\
& Z_{2} N_{2}
\end{array}\right] .
$$

3. Construct the matrices

$$
\hat{C}:=C \bar{V} \quad, \quad \hat{A}:=\bar{Z}^{T} A \bar{V} \quad, \quad \hat{B}:=\bar{Z}^{T} B .
$$

and define the reduced transfer function

$$
\hat{R}(s):=\hat{C}\left(s I_{2 n}-\hat{A}\right)^{-1} \hat{B} .
$$

From Theorem 6.5.1, $\hat{R}(s)$ is a second-order transfer function of order $n$ that satisfies the interpolation conditions (6.36). The algorithm above has al of the freedom in the method of forming the bases and selecting interpolation points and their associated orders found in the Rational Krylov family of algorithms [Gri97]. As a result, the second-order rational interpolation problem can be solved while exploiting the sparsity of the matrices and parallelism of the computing platform in a similar fashion.

\subsubsection{Second-order Structure Preserving Tangential Interpolation}

It is possible to generalize the earlier results for MIMO systems to perform tangential interpolation and preserve second-order structure. This is accomplished by replacing Krylov subspaces at each interpolation point, $\lambda_{i}$, with generalized Krylov subspaces as done in [GVV04a]. The spaces are defined as

Definition 6.5.2. Let $M \in \mathbb{C}^{n \times n}, X \in \mathbb{C}^{n \times m}, y^{[i]} \in \mathbb{C}^{m}, i=0, \ldots, k-1$ and define $Y \in \mathbb{C}^{k m \times k}$ as

$$
Y=\left[\begin{array}{ccc}
y^{[0]} & \ldots & y^{[k-1]} \\
& \ddots & \vdots \\
& & y^{[0]}
\end{array}\right] .
$$

A generalized Krylov subspace of order $k$, denoted $\mathcal{K}_{k}(M, X, Y)$, is the image of the matrix $\left[X M X \ldots M^{k-1} X\right] Y$.

For example, by using Algorithm 2 below to compute bases for generalized Krylov subspaces and forming the appropriate projections, one can construct a secorpl-order transfer function $\hat{R}(s)$ of order $n$ that satisfies the following

$$
x_{i}(R(s)-\hat{R}(s))=O\left(\lambda_{i}-s\right) \quad, \quad(R(s)-\hat{R}(s)) x_{i+n}=O\left(\lambda_{i+n}-s\right),
$$

where $x_{1}, \ldots, x_{n} \in \mathbb{C}^{1 \times p}$ and $x_{n+1}, \ldots, x_{2 n} \in \mathbb{C}^{m \times 1}$.

Algorithm 2 1. Construct $Z$ and $V$ such that 
$V=\left[\left(\lambda_{n+1} I_{2 N}-A\right)^{-1} B x_{n+1} \ldots\left(\lambda_{2 n} I_{2 n}-A\right)^{-1} B x_{2 n}\right]=\left[\begin{array}{l}V_{1} \\ V_{2}\end{array}\right]$ $Z^{T}=\left[\begin{array}{c}x_{1} C\left(\lambda_{1} I_{2 N}-A\right)^{-1} \\ \vdots \\ x_{n} C\left(\lambda_{n} I_{2 N}-A\right)^{-1}\end{array}\right]=\left[\begin{array}{ll}Z_{1}^{T} & Z_{2}^{T}\end{array}\right]$,

where $Z_{1}, Z_{2}, V_{1}, V_{2} \in \mathbb{C}^{N \times n}$. Choose the matrices $M_{1}, M_{2}, N_{1}, N_{2}$ $\mathbb{C}^{n \times n}$ such that $N_{1}^{T} Z_{1}^{T} V_{1} M_{1}=N_{2}^{T} Z_{2}^{T} V_{2} M_{2}=I_{n}$

2. Construct

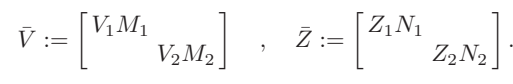

3. Construct the matrices

$$
\hat{C}:=C \bar{V} \quad, \quad \hat{A}:=\bar{Z}^{T} A \bar{V} \quad, \quad \hat{B}:=\bar{Z}^{T} B
$$

and define the reduced transfer function

$$
\hat{R}(s):=\hat{C}\left(s I_{2 n}-\hat{A}\right)^{-1} \hat{B} .
$$

It can be shown that $\hat{R}(s)$ is a second-order transfer function of order $n$ that satisfies the interpolation conditions (6.37) (see [GVV04a]).

It is also possible to impose higher order conditions while preserving the structure of the algorithm and the reduced order system. Consider, for instance, right tangential interpolation conditions of higher order (sim$x(s):=\sum_{i=0}^{k-1} x^{[i]}(s-\lambda)^{i}$. To impose the tangential interpolation condition

$$
(R(s)-\hat{R}(s)) x(s)=O(s-\lambda)^{k},
$$

we construct $\hat{R}(s)$ as in Algorithm 2 using the generalized Krylov subspace $\mathcal{K}\left((\lambda I-A)^{-1},(\lambda I-A)^{-1} B, X\right)$ where $X$ is formed from the $x^{[i]}, i=0, \ldots, k$

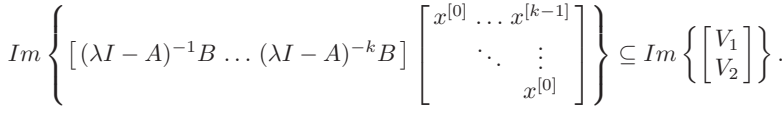

We refer to [GVV04a] for more details on this topic.

\subsection{Numerical Experiments}

In this section, model reduction techniques are applied to a large scale secondorder system repecting is to compare the performance of second-order structure preserving model reduction techniques, namely the SOBT technique introduced in Section 6.4 and the Second-Order Krylov technique introduced in Section 6.5, with respect to the standard first order techniques, namely the Balanced Truncation an the Multipoint Padé techniques.

The characteristics of the second-order system to be reduced are the following. The stiffness and mass matrix $S$ and $M$ are of dimension $N=26,394$. (See Chapter 24, Section 4, this volume, for a description of the example.) The mass matrix $M$ is diagonal and the stifness matrix $S$ is symmetric and

$$
C=B^{T}=[1 \ldots 1] .
$$

The damping matrix is proportional, meaning it is a linear combination of the mass matrix $M$ and the stiffness matrix $S$

$$
D:=\alpha M+\beta S,
$$

with $\alpha=0.675$ and $\beta=0.00315$. The second-order transfer function of McMillan degree $2 N=52788$ to be reduced is

$$
R(s):=B^{T}\left(s^{2} M+s D+S\right)^{-1} B=B^{T}\left(s^{2} M+s(\alpha M+\beta S)+S\right)^{-1} B .
$$

Given the structure of $M$ we normalize the equation so that the mass matrix is the identity as follows:

$$
\begin{gathered}
R(s)=B^{T} M^{-1 / 2}\left(s^{2} I+s\left(\alpha I+\beta M^{-1 / 2} S M^{-1 / 2}\right)+\right. \\
\left.M^{-1 / 2} S M^{-1 / 2}\right)^{-1} M^{-1 / 2} B
\end{gathered}
$$$$
:=\bar{C}\left(s^{2} I+s(\alpha I+\beta \bar{S})+\bar{S}\right)^{-1} \bar{B},
$$

where $\bar{S}:=M^{-1 / 2} S M^{-1 / 2}$ and $\bar{B}:=M^{-1 / 2} B=\bar{C}^{T}$

One intermediate system and five reduced order systems will be constructed from $R(s)$. Three reasons led us to construct an intermediate transfer function. First, concening the SVD techniques, it is not possible to apply the Balanced Truncation or the Second-Order Balanced Truncation methods directly to the transfer function $R(s)$ because its McMillan degree $2 N$ is too large for applying $O\left(N^{3}\right)$ algorithms. Second, the intermediate transfer function, assumed very close to $R(s)$, will also be used to approximate of the error bound between the diferent reduced transfer functions and the original transfer function $R(s)$. Finally, the intermediate transfer function will also be use in order to choose interpolation points for the Krylov techniques.

(i.e. of McMillan degree 400), called $R_{\text {a }}(\mathrm{s})$, is first constructed from $R(\mathrm{~s})$ using Modal Approximation by projecting $S$ onto its 
to its 200 eigenvalues of smallest magnitude. This corresponds to keeping the 400 eigenvalues of $s^{2} I+s(\alpha I+\beta S)+\bar{S}$ that are closest to the imaginary axis. Let $V_{f 200} \in \mathbb{R}^{2} \bar{S}$ the eigenvalues of $\bar{S}$ the closest to the imaginary axis (with $V_{f 200}^{T} V_{f 200}=I_{200}$ ) $\left(V_{f 200}\right.$ is computed with the Matlab function eigs). The intermediate transfer

$R_{200}(s):=\bar{C} V_{f 200}\left(s^{2} I+s\left(\alpha I+\beta V_{f 200}^{T} \bar{S} V_{f 200}\right)+V_{f 200}^{T} \bar{S} V_{f 200}\right)^{-1} V_{f 200}^{T} \bar{B}$.

By checking the difference between $R(s)$ and $R_{200}(s)$ at different points in the complex plane, it has been verified that the transfer functions are very close

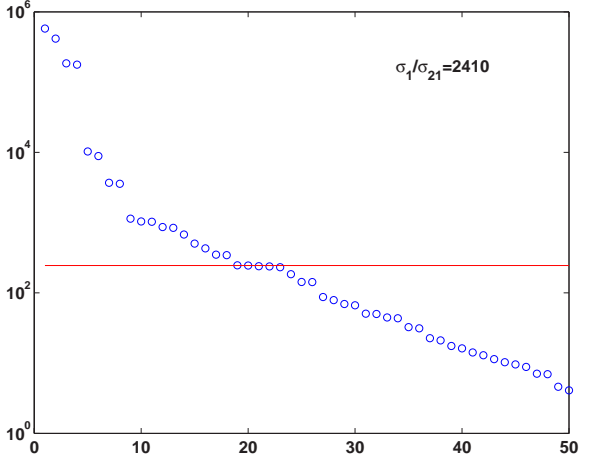

Fig. 6.1. Hankel singular values of $R_{200}(s)$

From $R_{200}(s)$, we compute the first reduced transfer function of McMillan degree 20 obtained by using balanced truncation (with the sysred Matlab function of the Niconet library), called $R_{b t}(s)$. Note that $R_{b t}(s)$ is no longer in second McMillan degree 40, callet

For the Krylov techniques, the reduced order transfer functions are computed directly from the original transfer function $R(s)$. Three reduced orde order Krylov procedure. The two other reduced systems (corresponding to different choices of interpolation points) are constructed using a second-order Krylov technique.
In order to apply Krylov techniques, a first important step consists in choosing the interpolation points. Indeed, the quality of the reduced order system is very sensitive to the choice of interpolation points.

An interesting fact is that there are 42 interpolation points between $R_{200}(s)$ and $R_{b t}(s)$ that have a positive real part (among the 420 zeros of $\left.R_{200}(s)-R_{b t}(s)\right)$. From several experiments, it has been observed that when using the standard Balanced Truncation technique, the number of interpolation points in the right-half plane between the original and the reduced transfer function is roughly equal to twice the McMillan degree of the rethe advantage that they are neither close to the poles of the plate he

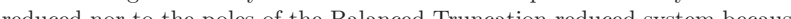
both tranfer function are stable. This implies that both tranfer functions

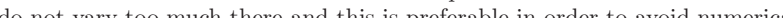
difficulties.

Because the McMillan degree of $R_{b t}(s)$ is equal to 20 , it is well known that 40 points are sufficient in order to describe $R_{b t}(s)$. In other words, the only transfer function of Mc Millan degree smaller than 20 that interpolates $R_{b s}(s)$ at 40 points in the complex plane is $R_{b t}(s)$ itself [GVV03]. So, we take the 40 interpolation points (these are 20 complex conjugate pairs of points) between $R_{200}(s)$ and $R_{b t}(s)$ with largest real part as our choice for computing the transfer function of McMillan degree 20, denoted $R_{K r y l}(s)$, that interpolates the original transfer function $R(s)$ at these points. The poles and interpolation points are shown in Figure 6.2. Because $R_{200}(s)$ is very close to $R(s), R_{\text {Kryl }}(s)$ should be close to $R_{b t}(s)$

Using the second-order Krylov technique, a reduced second-order transfer function $R_{\text {sokryl }}(s)$ of McMillan degree 28 is also constructed. Its McMillan degree was first chosen to be 20 but the resulting reduced transfer function was not stable. For this reason, additional interpolation conditions were added until the reduced transfer function was stable, resulting in a McMillan degre equal to 28 . The transfer function $R_{\text {sokryl }}(s)$ interpolates $R(s)$ at the 28 rightmost interpolation points between $R_{200}(s)$ and $R_{b t}(s)$.

For comparison purposes a set of interpolation points randomly generated (with symmetry with respect to the real axis in order to obtain a real interthe left baff plane $R_{2}(s)-R_{(0)}$ is alo These two sets of interpolation points are shown in Figure 6.3.

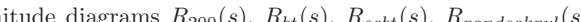
The $(s)$ and $R_{\text {sobry }}(s)$ are plotted in Figure 6.4. Recall, that $R_{200}(s)$ is used here as computationally tractable approximation of $R(s)$. More can be learned by considering the the $H_{-}$-norm errors relative to $\left\|R_{20}(s)\right\|$ shown in Table 6.1 .

As a first observation, it looks as if the six transfer functions are close to each other, especially for frequencies smaller than $10 \mathrm{rad} / \mathrm{sec}$ (where the bode 


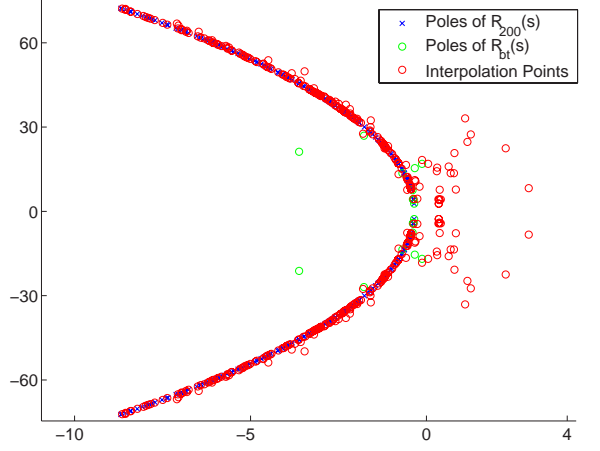

Fig. 6.2. Poles and interpolation points for $R_{200}(s)$ and $R_{b t}(s)$

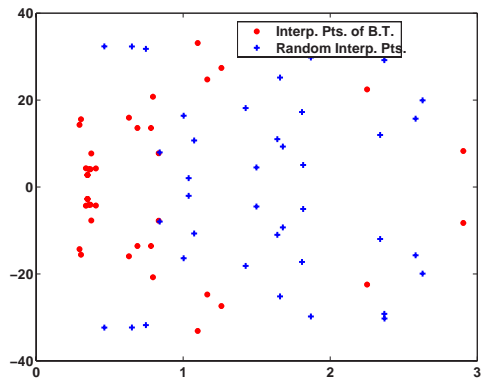

Fig. 6.3. Interpolation points for $R_{b t}(s), R_{\text {sokryl }}(s)$ and $R_{\text {randsokryl }}(s)$

magnitude diagrams are undistinguishable, see Figure 6.4). This is a good news because they should all approximate well the same transfer functio $R(s)$

One observes from Table 6.1 that the SVD techniques perform better than the Krylov techniques. Two remarks are in order. First, it should be kept in mind that only the Krylov reduced transfer functions are directly computed the the on the data of $R(s)$. Second, conching the Krylor techicher

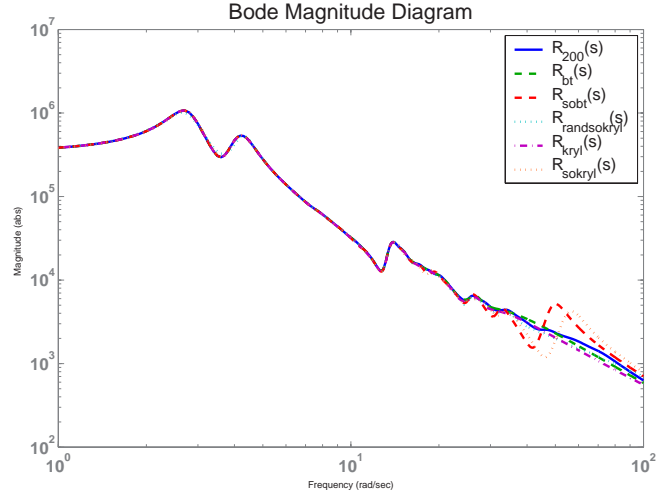

Fig. 6.4. The six transfer functions

Table 6.1. Relative errors for reduced order models

\begin{tabular}{llll}
\hline \multicolumn{2}{l}{$\begin{array}{l}\text { Reduced Transfer Model Reduction } \\
\text { function }\end{array}$} & technique & $\begin{array}{l}\text { McMillan } \\
\text { degree }\end{array}$ \\
\hline$R_{b t}(s)$ & Balanced Truncation & 20 & $4.310^{-4}$ \\
$R_{\text {sost }}(s)$ & Second-Order Bal. Trunc. & 40 & $2.610^{-4}$ \\
$R_{\text {kryl }}(s)$ & Krylov $(s)\left\|_{\infty}(s)\right\|_{\infty}$ \\
$R_{\text {sokryl }}(s)$ & Second-Order Krylov & 20 & $8.310^{-4}$ \\
$R_{\text {randsokryl }}(s)$ & Random Second-Order Krylov 20 & $5.810^{-2}$ \\
\hline
\end{tabular}

polation points. Because for SISO systems, any transfer function can be constructed from Krylov subspaces from any transfer function of larger McMillan degree, there should exist interpolation conditions that produce reduced order transfer functions with smaller error bound than what can be obtained with balanced tech conditions.

cerning SVD techniques is that the best approximation is obtained with $R_{s o b t}(s)$ and not $R_{b t}(s)$. Nevertheless, one should not forget that the McMillan degree of $R_{\text {sobt }}(s)$ is twice as large as the McMillan degree of $R_{b t}(s)$.

In contrast with SVD techniques, the error obtained with the first order transfer function $R_{k r y l}(s)$ is 100 times smaller than for the second-order Second-Order Krylov techniques perform quite poorly compared to the first 
order techniques, perhaps indicating that a more sophisticated algorithm for choosing the interpolation points for these methods is needed.

Finally, by choosing random interpolation points, the error remains roughly the same than by taking the balanced truncation interpolation points: 0.058 for $R_{\text {sokryl }}(s)$ and 0.07 for $R_{\text {randsokryl }}(s)$. This is probably due to the fact that the area chosen to generate the interpolation points for $R_{\text {randsokryl }}(s)$

\subsection{Concluding Remarks}

Concerning the second-order Krylov technique, the following observation is worth mentioning. For SISO systems of pair Mc Millan degree, it has been shown in [BSGLO4] and $[\mathrm{NS}$ s that for every first order system $(c, A, b)$ suc second-order form In other words, every SISO system (with fist Martor parameter equal to zero) can be rewritten as a second-order system. Thi implies that in the SISO case, it is possible to impose $4 n-1$ interpolation conditions for a reduced second-order system of McMillan degree $2 n$ by first using the standard Multipoint Padé technique of Theorem 6.2.3 and then reconstructing a second-order form with an appropriate state space coordinate transformation. Currently, no proof is available for the MIMO case.

As for generalized state space realizations of first order systems, it is also possible to apply Krylov techniques to second-order systems without requiring the mass matrix $M$ to be equal to the identity. Concerning the SOBT technique, special care must taken in deriving the second-order Gramians

For second-order balanced truncation, numerical results are very encouraging, but many important questions remain open. For instance, does there exist an a priori global error bound with SOBT, as for Balanced Truncation? Even simpler, is stability of the reduced system always guaranteed? If the answer to the preceding questions is negative, does there exist a better choice applicable to large scale systems is needed.

\section{Acknowledgement}

This paper presents research supported by NSF grants CCR-99-12415 and ACI-03-24944, and by the Belgian Programme on Inter-university Poles of Technology and Culture. The scientific responsibility rests with its authors.

\section{References}

[Ant05] Antoulas, A.: Lectures on the Approximation of Large-scale Dynamical Systems. SIAM, Philadelphia, to appear (2005)

[BS04] Z Bai and Y Su. Dimension reduction of second order dynamical system via a second-order arnoldi method. Technical Report CSE-2004-1, University of California, Davis, 2004

[BSGL04] Bunse-Gerstner, A., Salimbahrami, B., Grotmaack, R. and Lohmann, B. textence and Computation of Second Order Reduced Systems using Krylo Subspace Methods. In: Proceedings of 16th Symp. on the Mathematical The

ory of Networks and Systems, Leuven (2004)
[CGV04] Chahlaoui, Y., Gallivan, K. and Van Dooren, P.: The $H_{\infty}$ norm calculation or large sparse systems lin: Proccalings of 16th Symp. on the

[CLVV05] Chahlaoui, Y., Lemonnier, D., Vandendorpe, A. and Van Dooren, P.: Second-order
appear $(2005)$

[dVS87] de Villemagne, C. and Skelton, R.: Model reductions using a projection formulation. Int. J. Control, 46, 2141-2169 (1987)

jection Methods for Model Reduction. PhD thesis, University of Illinois, Urbana-Champaign, (1997)

Doren, P. Model Reduction via runcation : an interpolation point of view. Linear Algebra and its Applica(2003)

[GVV04a] Gallivan, K., Vandendorpe, A. and Van Dooren, P.: Model reduction a

Analysis and Applications, 26(2), 328-349 (2004)
b] Gallivan. K., Vandendorpe, A. and Van Dooren. P.: Svlvester equations and projection-based model reduction. Journal of Computational and $A p-$ 162, 213-229 (2004)

[MS96] Meyer, D. and Srinivasan, S.: Balancing and model reduction for secondDinar systems. IEEE Trans. Automat. Control, 41(11), 1632$1644(1996)$

[Pre97] Preumont, A.: Vibration Control of Active Structures. Kluwer Academic Publishers, Dordrecht (1997)

[SA02] Sorensen, D. and Antoulas, A.: The Sylvester equation and approximate s, 351-352, 671-700

[SC91] Su, T. and Craig, J.: Model reduction and control of flexible structures using krylov vectors. J. Guidance Control Dynamics, 14(2), 1311-1313 (1991)

V04] Vandendorpe, A. and Van Dooren, P.: Krylov techniques for model reduc-
tion of second-order systems. Int. Rept. CESAME TR07-2004, Universite catholique de Louvain, Louvain-la-Neuve (2004)

[WJJ87] Weaver, W. and Johnston, P.: Structural Dynamics by Finite Element.

Prentice Hall, Upper Saddle River (1987)
[ZDG95] Zhou, K., Doyle, J. and Glover, K. Robust and Optimal Control. Prentice Hall, Upper Saddle River (1995) 